\title{
Brain Edema: Newer Concept of Treatment
}

\author{
${ }^{1}$ Department of Neurosurgery, Institute of Medical Science and \\ SUM Hospital, Bhubaneswar, Odisha, India
}

Sureswar Mohanty ${ }^{1}$ Srikant Kumar Swain ${ }^{1} \quad$ Chinmay Biswal ${ }^{1}$

\begin{abstract}
Address for correspondence Sureswar Mohanty, MS, MCh, FAMS, FACS, Department of Neurosurgery, Institute of Medical Science and SUM Hospital, Bhubaneswar, Odisha 751003, India (e-mail: sureswar.mohanty@gmail.com).
\end{abstract}

\begin{abstract}
Brain edema is excess accumulation of water in intracellular or extracellular spaces of the brain. It may be due to traumatic brain injury, neoplasm, infection, or following surgery. Advent of electron microscope and molecular pathophysiology of fluid transport through blood-brain barrier has elucidated the mechanism of edema formation, that is, ion channels and transport of fluid into extracellular space. Currently approved treatments, such as decompressive craniectomy and osmotherapy, controlled hyperventilation, and administration of diuretics, were developed prior to any knowledge of modern cerebral edema pathophysiology. These therapies attempt to manage downstream end-stage events without directly attenuating the underlying molecular mechanisms of cerebral edema. Next few years will yield new knowledge of how particular proteins drive edema influx, paving the way for rationally designed therapeutics that directly target key steps in cerebral edema formation, thereby achieving what currently approved therapies do not. Pharmacological agents which can block edema formation are being tried experimentally and clinically. Development in imaging, that is,

Keywords

- cerebral edema

- blood-brain barrier

- inflammation

- corticosteroid

- ion transport computed tomography and diffusion tensor magnetic resonance imaging, has helped in antemortem assessment of evolution and resolution of brain edema as a dynamic pathophysiology. Animal studies shows release of vasoactive substances, that is, histamine, serotonin, adrenaline, nitric oxide, substance $P$, prostaglandins, tumor necrosis factor- $\alpha$, and cytokines, in the injured brain results in activation of inflammatory cascade, which is the important cause of brain edema.
\end{abstract}

\section{Introduction}

Brain edema is an excess accumulation of water in the intracellular and/or extracellular spaces of the brain. Brain edema after traumatic brain injury is a frequent finding. Brain edema is often associated with neoplasm, infection, that is, abscess and granulomas, and following surgery. Grossly, in older German terminology of brain edema, the cut surface oozes fluid (Hirn edem). In brain swelling, the cut surface is dry (Hirn swellung). With the advent of light and electron microscope and molecular basis of fluid transport through blood-brain barrier (BBB), further understanding of the structure and function of the barrier and the mechanism of edema formation has evolved in the last few decades, that is, ion channels and transports, so pharmacological agents which can block edema formation is being tried experimentally and clinically. ${ }^{1,2}$ New developments in imaging, that is, computed tomography and diffusion tensor magnetic resonance imaging, has helped in antemortem diagnosis, evolution, and resolution of brain edema as a dynamic pathophysiology. ${ }^{3,4}$

\section{Types of Brain Edema}

Vasogenic brain edema is caused by disruption of the BBB. Intravascular fluid escapes through the endothelium (pinocytosis), or leaky capillary tight junction, for example, through trauma, tumor, hemorrhage, and granuloma.

Cytotoxic edema (oncotic cell swelling) is characterized by accumulation of water inside the neurons, microglia, and astrocytes. Sometimes they bloat or rupture and fluid 
escapes into extracellular space, for example, infarction and neurotoxic agents. Ionic edema, an extracellular edema that occurs in the presence of an intact BBB, forms immediately following cytotoxic edema.

Osmotic edema occurs when plasma dilution decreases serum osmolality, resulting in a higher osmolality in the brain compared with the serum. This creates an abnormal pressure gradient and movement of water into the brain, which can cause progressive cerebral edema, resulting in a spectrum of signs and symptoms from headache and ataxia to seizures and coma, for example, water intoxication and hepatic failure.

Hydrocephalic edema (interstitial) occurs in obstructive hydrocephalus due to a rupture of the cerebrospinal fluid (CSF)-brain barrier. This results in transependymal flow of CSF, causing CSF to penetrate the brain and spread to the extracellular spaces and the white matter. Interstitial cerebral edema differs from vasogenic edema as CSF contains almost no protein.

\section{Experimental/Clinical Studies}

Since the release of vasoactive chemicals in the injured tissue of live human being is difficult to demonstrate, we used animal head injury models by direct demonstration of vasoactive substances done in the injured brain. Samples of edematous brain were collected during surgery, and blood samples of head injured patients were collected serially demonstrating rise of vasoactive substances. Prognosis following head injury patients correlated well with the decrease and normalization of vasoactive agents. Interestingly, immunological study in severely head injured patients shows rebuilt of antibrain antibodies possibly due to escape of cerebroproteins to circulation. . $^{-10}$

Inflammatory substances mediating vasogenic brain edema has been studied in rats, dogs, rabbits, and cats. Trauma was produced by stab wound of the brain in the former and fall of weight in the later animals. Intracerebral hemorrhage was created by injecting blood into the brain of rats. ${ }^{8}$ Important finding was the release of vasoactive substances resulting in inflammation, that is, histamine, serotonin, adrenaline, nitric oxide, substance $P$, prostaglandins, tumor necrosis factor- $\alpha \subseteq$ and cytokines, in the injured brain. ${ }^{5-8,10}$ Recent studies reveal important role of inflammation as a cause of edema formation. ${ }^{7,11}$ All those substances play an important role in opening the endothelial junction and efflux of fluid, leukocytes, and platelet into the extracellular space. They also help in the escape of intravascular fluid through endothelial cells by pinocytosis. Increased levels of biogenic amines and tissue enzymes in the blood/CSF is an indirect evidence of increased BBB permeability and related to prognosis. ${ }^{12-19}$

\section{Pathogenesis}

Disruption of the BBB is the most important prerequisite for edema formation. Several mediators have been discovered to act at the BBB either passively or actively. Serum which escapes into the extracellular spaces ultimately increase tissue volume and raise intracranial pressure (ICP). Both vasogenic and cytotoxic edema results in increased ICP and eventually decreased cerebral perfusion pressure (CPP). This is in line with the Monro-Kellie hypothesis which states that "the sum of the intracranial volumes of blood, brain, CSF, and other components is constant and that an increase in any one of these must be offset by an equal decrease in another." Elevated ICP and diminished cerebral perfusion can lead to tissue ischemia. Ischemia in turn activates autoregulatory mechanisms designed to restore cerebral perfusion. However, vasodilation increases cerebral blood volume, which in turn increases ICP, lower CPP, and provokes further ischemia. ${ }^{17}$

After traumatic brain injury, cerebral blood flow (CBF) autoregulation is impaired or abolished in most patients. When pressure autoregulation is impaired or absent, ICP decreases and increases with change in CPP. Also, autoregulatory vasoconstriction seems to be more resistant compared with autoregulatory vasodilation which indicated that patients are more sensitive to damage from low rather than high CPPs. Molecular biologic studies recently reveals trance endothelial passage of fluid into the extracellular space resulting in brain edema by active transporter and aquaporins. ${ }^{2,12}$

\section{Pitfalls of Using Animal Models (Factors of Laboratory to Bedside Treatment Translational)}

Commonly used animal model of acute central nervous system injury do not accurately reflect human disease. Fault with experimental design may lead to false positives. Clinical trials often do not replicate promising results in experimental studies.

\section{Treatment of Brain Edema}

The goal of medical management of cerebral edema is to maintain optimal ICP, ensure regional and global CBF to meet the metabolic requirements of the brain, and prevent secondary neuronal injury from cerebral ischemia. ${ }^{17}$

Standard medical management of cerebral edema involves using a systemic approach, from general measures, that is, optimal head and neck positioning for facilitating intracranial venous outflow, proper airway, avoidance of dehydration, and systemic hypotension, and maintenance of normothermia, to specific therapeutic interventions like controlled hyperventilation, administration of diuretics, osmotherapy, and pharmacological cerebral metabolic suppression. ${ }^{17,20,21}$ Some of the drugs clinically used and others under experimental studies are listed in - Table $\mathbf{1}$.

Future treatment is possibility of drug cocktail which will be useful to prevent secondary brain injury and protect the neurons.

Comparison of vasogenic edema associated with trauma and tumor:Dexamethasone and mannitol are very effective in cases of tumor edema ( Figs. 1 and $\mathbf{2}$ ), ${ }^{4,17}$ whereas it is not very effective in trauma edema. Thus, traumatic vasogenic edema is multifactorial involving BBB leakage, cytoplasmic 
Table 1 Drugs reducing vasogenic brain edema

\begin{tabular}{|c|c|}
\hline Factors increasing edema & Inhibitory/Blocking substances \\
\hline $\begin{array}{l}\text { Ion channel cotransporter- } \mathrm{Na}^{+}-\mathrm{K}+-2 \mathrm{Cl} \text { - cotransporter } \\
\text { suri regulator NC C8-ATP }\end{array}$ & $\begin{array}{l}\text { Bumetanide } \\
\text { Glibenclamide }\end{array}$ \\
\hline - Vasopressin (V1 A and V2 receptor) antagonist & Conivaptan \\
\hline $\begin{array}{l}\text { - Inflammation mediators } \\
\text { - Oxidative mediators } \\
\text { - Adhesion mediators } \\
\text { - Cytokines, IL-1 } \alpha, 1 \beta, \text { TNF } \alpha, \text { IL-6 } \\
\text { - Chemokines } \\
\text { - Catecholamines }\end{array}$ & $\begin{array}{l}\text { Anti-inflammatory drugs, i.e., indomethacin } \\
\text { steroids } \\
\text { Pentoxifylline } \\
\text { pCPA } \\
\text { H2-blockers-ranitidine/cimetidine } \\
\text { Ibuprofen }\end{array}$ \\
\hline $\begin{array}{l}\text { - Enzymes (increases blood and CSF) } \\
\text { LDH, ALD, MDH, GPT, GOT, cyclooxygenase-2 }\end{array}$ & $\begin{array}{l}\text { Cortisone, CRF } \\
\text { Metaraminol } \\
\text { Acetazolamide } \\
\text { Dextran } \\
\text { Urea } \\
\text { SC236 and dexamethasone rofecoxib }\end{array}$ \\
\hline - Hemoglobin degradation product(free iron) & Iron chelation \\
\hline - Free fatty acids & Endogenous inhibitors (long chain fatty acids) \\
\hline $\begin{array}{l}\text { - Prostaglandins mitochondrial permeability dam- } \\
\text { age cerebral anaerobic metabolism polyamines free } \\
\text { radicals } \\
\text { endothelin } \\
\text { chloride transport carbonic anhydrase kappa opioid } \\
\text { aquaporin } 4\end{array}$ & $\begin{array}{l}\text { Indomethacin } \\
\text { Cyclosporin A } \\
\text { Citicoline lactate } \\
\text { NMDA receptor antagonists-ifenprodil } \\
\text { Scavengers-vitamin C and E } 21 \text { aminosteroids } \\
\text { Edaravone } \\
\mathrm{N} \text {-acetyl cysteine } \\
\text { Citicholine } \\
\text { Endothelin antagonists-patent EPO } 838223 \mathrm{CL} \text { transport inhibitor-torase } \\
\text { CA inhibitors-acetazolamide agonist, niravoline, dexamethasone, and HCRF }\end{array}$ \\
\hline
\end{tabular}

Abbreviations: CA, carbonic anhydrase; CL, chloride; CRF, corticotropin-releasing factor; HCRF, human corticotropin-releasing factor; IL, interleukin; TNF, tumor necrosis factor.
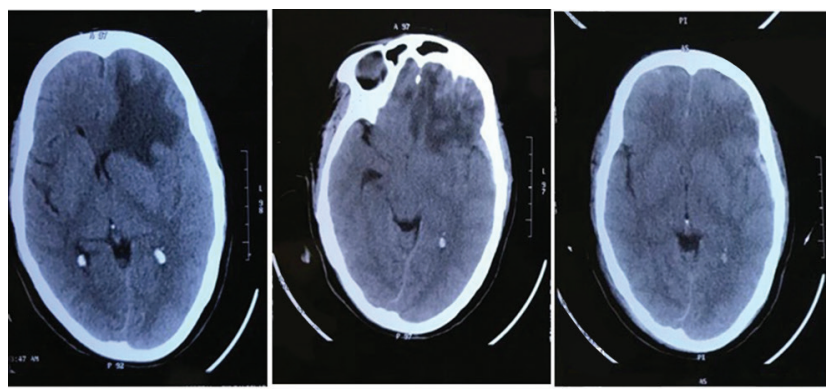

Fig. 1 Resolution of traumatic edema (A-C). (A) Postinjury. (B) 72 hours later (20\% mannitol intravenously $100 \mathrm{~mL} / 8$ hours). (C) 2 weeks later (no intravenous mannitol).
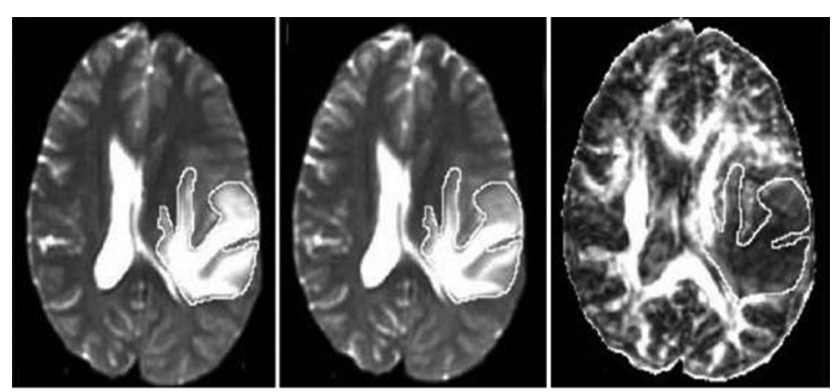

Fig. 2 (A-C)Resolution of peritumoral edema (diffusion tensor magnetic resonance imaging [DT-MRI]) with dexamethasone treatment. (A) Before steroid therapy. (B) 24 hours after steroid therapy. (C) 72 hours after steroid therapy. transport, inflammatory mediators released from endothelium, platelets, and glial cells.

Surgical decompression and use of osmotherapy to reduce brain edema and its deleterious effect remains the mainstay of treatment even today. This only attenuates the primary injury but cannot abate the secondary cascade of events. Drugs which inhibit or slow the various secondary mechanisms are still in an experimental stage.

\section{Conclusion}

Currently approved treatments for cerebral edema-decompressive craniectomy and osmotherapy-were developed prior to any knowledge of modern cerebral edema pathophysiology. These therapies attempt to manage downstream end-stage events without directly attenuating the underlying molecular mechanisms of cerebral edema. ${ }^{2}$

The water movements involved in cerebral edema are dependent upon ionic fluxes, which are ultimately mediated by individual channels and transporters. The study of cerebral edema is essentially the study of maladaptive ion transport. While significant gaps still remain in our understanding of how specific proteins contribute to cerebral edema, the fields of cerebral edema and brain CSF dynamics are robust and productive. Doubtlessly, the next few years will yield new knowledge of how particular proteins drive edema 
influx, paving the way for rationally designed therapeutics that directly target key steps in cerebral edema formation, thereby achieving what currently approved therapies do not.

\section{Conflict of Interest}

None declared.

\section{References}

1 Patro A, Mohanty S. Pathophysiology and treatment of traumatic brain edema. Ind J Neurotrauma 2009;6:11-16

2 Stokum JA, Gerzanich V, Simard JM. Molecular pathophysiology of cerebral edema. J Cereb Blood Flow Metab 2016;36(3):513-538

3 Klatzo I. Presidental address. Neuropathological aspects of brain edema. J Neuropathol Exp Neurol 1967;26(1):1-14

4 Sinha S, Bastin ME, Wardlaw JM, Armitage PA, Whittle IR. Effects of dexamethasone on peritumoural oedematous brain: a DT-MRI study. J Neurol Neurosurg Psychiatry 2004;75(11):1632-1635

5 Mohanty S, Mazumdar S. Role of serotonin in human cerebral oedema and contusion. Indian J Med Res 1978;67:1029-1032

6 Nayak AK, Mohanty S, Singh RK, Chansouria JP. Plasma biogenic amines in head injury. J Neurol Sci 1980;47(2):211-219

7 Mohanty S, Ray AK, Dey PK. Cerebral oedema and blood-brain and blood-CSF barriers in experimental brain trauma: effect of indomethacin-A prostaglandin synthetase inhibitor. Indian J Physiol Pharmacol 1980;24(2):91-96

8 Mohanty S. Role of monoamine neurotransmitters in pathophysiology of head injury. Icmr Bull 1980;10:167

9 Bhattacharya RN, Mohanty S, Mukherjee KC, et al. Antibrain antibodies in brain tumour. Clinician (Goa) 1979;43:1

10 Butcher K. Inflammation in intracerebral hemorrhage: clearly present, but what is its role? Neurol India 2006;54(4):352-353
11 Himadri P, Kumari SS, Chitharanjan M, Dhananjay S. Role of oxidative stress and inflammation in hypoxia-induced cerebral edema: a molecular approach. High Alt Med Biol 2010;11(3):231-244

12 Qing WG, Dong YQ Ping TQ et al. Brain edema after intracerebral hemorrhage in rats: the role of iron overload and aquaporin 4. J Neurosurg 2009;110(3):462-468

13 Mohanty S, Rao CJ, Nayak AK, et al. Significance of certain plasma enzymes and biogenic amines in head injury. Seara Med Neuro Civ 1982;11:7-16

14 Mohanty S, Dey PK, Sharma HS, Singh S, Chansouria JP, Olsson Y. Role of histamine in traumatic brain edema. An experimental study in the rat. J Neurol Sci 1989;90(1):87-97

15 Mohanty S, Bishnu PP, Tandon SC. Significance of plasma histamine levels in head injury. Neurol India 1990;38:117-124

16 Patnaik R, Mohanty S, Sharma HS. Blockade of histamine H2 receptors attenuate blood-brain barrier permeability, cerebral blood flow disturbances, edema formation and cell reactions following hyperthermic brain injury in the rat. Acta Neurochir Suppl (Wien) 2000;76:535-539

17 Rasmussen T, Gulati DR. Cortisone in the treatment of postoperative cerebral edema. J Neurosurg 1962;19:535-544

18 Rao CJ, Shukla PK, Mohanty S, Reddy YJ. Predictive value of serum lactate dehydrogenase in head injury. J Neurol Neurosurg Psychiatry 1978;41(10):948-953

19 Rao CJ, Mohanty S, Shukla PK, Reddy YJV. Significance of serum cholinesterase levels in human head injury. Indian J Med Res 1978;68:668-674

20 Raslan A, Bhardwaj A. Medical management of cerebral edema. Neurosurg Focus 2007;22(5):E12

21 Turel MK, Moorthy RK, Sam GA, et al Effect of pretreatment with a tyrosine kinase inhibitor (PP1) on brain oedema and neurological function in an automated cortical cryoinjury model in mice. J Clin Neurosci 2013;20(4):593-596 